\title{
The clinical significance of tumor-infiltrating neutrophils and neutrophil-to-CD8+ lymphocyte ratio in patients with resectable esophageal squamous cell carcinoma
}

\author{
Jianbo Wang, Yibin Jia, Nana Wang, Xiaomei Zhang, Bingxu Tan, Guangyu Zhang and Yufeng Cheng*
}

\begin{abstract}
Background: The interaction between tumor cells and inflammatory cells has not been systematically investigated in esophageal squamous cell carcinoma (ESCC). The main aims of the study were to investigate the clinical significance of tumor-infiltrating neutrophils and neturophil-to-CD8+ lymphocyte ratio (NLR), and to analyze the distribution of tumor-infiltrating neutrophils and CD8+ lymphocytes in ESCC treated by curative resection.

Methods: The expressions of CD66b and CD8 were assessed with double staining immunohistochemistry in the surgical specimens from 90 patients with ESCC treated by curative surgery.

Results: We showed that increased intratumoral neutrophils were significantly associated with lymph node metastasis $(P=0.016)$, and advanced pathological stages $(P=0.013)$. Decreased peritumoral $C D 8+$ lymphocyte density was more frequently observed in patients with single positive lymph node $(p=0.045)$. Peritumoral NLR was significantly associated with advanced T stages $(p<0.001)$, lymph node metastasis $(p=0.041)$ and a trend towards advanced pathological stages $(p=0.053)$. Increased intratumoral neutrophils were significantly associated with decreased disease-free survival $(p<0.001)$ and overall survival $(p<0.001)$ in univariate analysis and were identified as an independent prognostic factor for disease-free survival $(p=0.006)$ and overall survival $(p=0.037)$ in multivariate analysis. Neither the density nor the distribution of tumor-infiltrating neutrophils was significantly correlated with that of CD8+ lymphocytes. The density of intratumoral CD8+ lymphocytes was significantly lower than $(P<0.001)$ and moderately correlated with $(r=0.434$, $p<0.001)$ that in peritumoral area.

Conclusions: Increased intratumoral neutrophils were an independent poor prognostic factor and peritumoral NLR was significantly associated with disease progression in ESCC treated by curative surgery, suggesting the possible effect of immune misbalance of tumor microenvironment in facilitating ESCC progression. Immunotherapy targeted to the above predictors should be considered in the future.
\end{abstract}

Keywords: Intratumoral neutrophils, Neturophil-to-CD8+ lymphocyte ratio, Esophageal squamous cell carcinoma, Immunohistochemistry, Microenvironment

\section{Background}

Esophageal cancer is the eighth most common cancer type and the sixth leading cause of cancer death worldwide, which was responsible for 482300 new cases and 406800 deaths in 2008 [1]. In China, esophageal cancer represents a major health problem, which is the fourth

\footnotetext{
*Correspondence: qlcyf1965@163.com

Department of Radiation, Qilu Hospital of Shandong University, Jinan 250012, P.R. China
}

leading cause of cancer death. Squamous cell carcinoma accounts for more than $90 \%$ of esophageal cancer cases in Chinese patients [2]. Despite the advancement in diagnosis and treatment modalities, esophageal squamous cell carcinoma (ESCC) still shows a dismal prognosis with a 5-year survival rate less than 15\% [3,4]. The current prognostic model is mainly based on pathological parameters such as histological subtype, tumor size and pathological tumor-node-metastasis (pTNM) classification, which are 
urgently needed to be improved by the integration of new prognostic biomarkers.

Immune reaction was proposed as the seventh hallmark of cancer [5]. The importance of interaction between neoplastic cells and inflammatory cells is becoming increasingly recognized [6]. Leukocyte infiltration is one main characteristic of almost all malignant tumors and the major constituents of these infiltrates include tumorassociated macrophages, neutrophils, mast cells, NK cells, lymphocytes, and so on, but the prognostic value of these infiltrates is still controversial.

Neutrophils, which represent $50 \%-70 \%$ fraction of total circulating leukocytes, make up a significant portion of the leukocyte infiltration in a wide variety of human cancers [7]. Although commonly encountered within tumor microenvironment, neutrophils have not been traditionally considered as a disease modifying entity. Many type of cells within the tumor microenvironment are capable of secreting neutrophil chemotactic substances and neutrophils recruited to tumor site seem to promote cancer cell migration and invasion [8]. Increasing evidence indicated that the presence of neutrophils in tumor tissue be associated with poor prognosis [9-11]. Jensen and colleagues [9] examined the infiltration of neutrophils in 121 localized renal cell carcinomas and showed that the presence of intratumoral neutrophils is an independent poor prognostic factor. Rao et al. [11] provided evidence that increased intratumoral neutrophils be important in the acquisition of a malignant phenotype and predict adverse prognosis in colorectal carcinomas. However, until now, the prognostic effect of tumor-infiltrating neutrophils in ESCC remains unknown.

An elevation in blood neutrophil-to-lymphocyte ratio is considered as a marker of systemic inflammation, which predisposes the tumor to proliferate and metastasize through inhibition of apoptosis, promotion of angiogenesis, and damage of DNA [12-14]. Recent evidence has shown that a high preoperative blood neutrophil-to-lymphocyte ratio was associated with poor outcome in various malignancies undergone potentially curative resection, including esophageal cancer $[15,16]$. However, the interaction between immune system and tumor cells mostly take place around the tumor tissue. The neutrophil-to-lymphocyte ratio in tumor tissue may also serve as an immune marker of prognostic significance. Cytotoxic CD8+ lymphocytes are crucial components of tumor-specific cellular adaptive immunity and constitute the predominant lymphocyte infiltration in tumor tissue. It is necessary to study the clinical significance of neturophil-to-CD8 + lymphocyte ratio (NLR) in tumor tissue. To date, the prognostic effect of tumor-infiltrating NLR has not been investigated in ESCC.

The main aims of the study were to investigate the clinical significance of tumor-infiltrating (intratumoral and peritumoral) neutrophils and NLR, and to analyze the distribution of tumor-infiltrating neutrophils and CD8+ lymphocytes in ESCC treated by curative resection. The prognostic effect of tumor-infiltrating CD8+ lymphocytes was also studied.

\section{Materials and methods \\ Patients and specimens}

Between January 1st and December 31st 2007, 90 patients, who underwent potential radical surgery for ESCC in Department of Thoracic Surgery at Qilu Hospital of Shandong University, were included in the study. Patients were already excluded because of neoadjuvant treatment, perioperative mortality, distant metastasis, stage 0 disease, resection not for curative intent, lost to follow up and lack of tumor tissue. The protocol of the study was approved by the Institutional Ethic Committee of Qilu Hospital of Shandong University. All the patient demographic and clinical data including age, gender, histological grade, lymph node status, pTNM stage, and adjuvant treatment were abstracted from the clinical records. Tumor staging was based on the American Joint Committee on Cancer (AJCC) $6^{\text {th }}$ edition staging manual [17]. A thorough histological examination was made using $\mathrm{H} \& \mathrm{E}$-stained tissue preparation and the histological grade was determined according to the degree of differentiation of the tumor. Follow-up visits were performed every 3 months for the first 2 years after surgery and thereafter every 6 months up to death or the end of the study (December 2012) for patients without death. At each visit, a clinical history was taken and a physical examination was performed. Routing diagnostic imaging methods included barium meal fluoroscopy and computer tomography. Disease-free survival (DFS) was measured from the date of operation to the date of first evidence of relapse or death, whichever was observed first. For patients who had not relapsed or died, DFS was censored at the last date that the absence of relapse was confirmed. Overall survival (OS) was measured from the date of surgery to the date of death or last followup for surviving patients [18].

\section{Immunohistochemistry}

Formalin-fixed paraffin-embedded surgical specimens were used for the immunohistochemical analysis. The presence of available tumor was confirmed by hematoxylin and eosin staining. The tissue blocks were sectioned at $2 \mu \mathrm{m}$ and mounted on glass slides. Primary antibodies were against CD8 (clone C8/144B, diluted at 1:100, DAKO Corporation Carpenteria, CA, USA) and CD66b (clone G10F5, diluted at 1:200, BD Biosciences, San Jose, CA, USA). Double staining of CD66 and CD8 was carried out with DouSP KIT (Maixin-bio, Jinan, China) to analyze the densities and distributions of CD66+ neutrophils and CD8+ lymphocytes following the manufacturer's protocol with some 
modification. The sections were deparaffinized in xylene and rehydrated through a graded ethanol series. Antigen retrieval was performed with $0.01 \mathrm{M}$ citrate buffer $(\mathrm{pH}$ 6.0) in a pressure cooker for $3 \mathrm{~min}$. Then endogenous peroxidase activity was blocked with endogenous peroxidase blocking solution and non-specific protein-binding sites were blocked through $20 \mathrm{~min}$ incubation with nonimmune serum at room temperature.

The anti-CD8 antibody was applied for at $37^{\circ} \mathrm{C}$ for $1.5 \mathrm{~h}$ followed by three 5-min washes with PBS. After incubated with biotinylated-labeled second antibody for $30 \mathrm{~min}$, slides were rinsed three times in PBS for $5 \mathrm{~min}$ and then incubated in streptavidin alkaline phosphatase complex. After color development with the BCIP/NBT reagent, the sections were washed with PBS, followed by the double-stain amplifier provided with the kit. The antiCD66b primary antibody was applied at room temperature for $1.5 \mathrm{~h}$, and washed with PBS. Then, the biotinylatedlabled second antibody was applied for 30 min followed by streptavidin peroxidase. The immunohistochemical staining was visualized with AEC reagent. Finally, the sections were stained with hematoxylin for $1 \mathrm{~min}$ and mounted with an aqueous mounting medium supplied with the kit.

\section{Evaluation of immunostaining}

The analysis was performed with an Olympus IX71S1F-3 Inverted Microscope by two independent observers (Yibin Jia and Nana Wang) blinded to the patients' clinicopathological details. The average of the values obtained by the two observers was recorded. Intratumoral leukocytes were determined as neutrophils or CD8+ lymphocytes that infiltrated into cancer nests or stroma, whereas peritumoral leukocytes were the cells that distributed along the tumor-myometrial or tumor-connective tissue junction. The tumor sections were screened at low power magnification $(\times 100)$, and 6 high power fields $(\times 400)$ with highest number of cell infiltration in either intratumoral or peritumoral areas for neutrophils or CD8+ lymphocytes were selected. The median leukocyte count was used as cutoff to categorize each case into either a high or low group. Areas of necrosis and artifacts and cells within the vessels were omitted. The intratumoral or peritumoral NLR was calculated according to the method of one previous study [19]. NLR was categorized as either $\geq$ or $<1$.

\section{Statistical analysis}

Statistical analyses were performed with the SPSS statistical software package (SPSS version 13.0; Chicago, IL). Differences between groups were compared using the chi-square test for categorical variables and the Student $t$ test for continuous variables. Associations between continuous variables were examined by calculating Pearson's correlation coefficient. For univariate analysis, survival curves were obtained with the Kaplan-Meier method and compared by log-rank test. The Cox proportional hazard regression model was used to identify independent prognostic factors. All statistical tests were 2 -sided, and P values $<0.05$ indicated of statistical significance.

\section{Results}

\section{Patient characteristics}

Part of the patient characteristics are listed in Table 1. Median age was 60.5 years (range, 42 to 78 years), and $80 \%$ of patients were male. Tumor locations were upper thoracic in 10 patients, middle thoracic in 48 patients, and lower thoracic in 32 patients. The median length of the tumor was $4 \mathrm{~cm}$ (range, 0.5-8.5 cm). The histopathological differentiations were poor in 26 cases, moderate in 39 cases, and well in 25 cases. 59 patients $(65.6 \%)$ had T3/T4 tumors. 33 patients (36.7\%) have positive lymph nodes. The pathological stages were stage I in 18 patients, stage II in 41 patients and stage III in 31 patients. 55 patients $(61.1 \%)$ received surgery alone, 10 (11.1\%) received postoperative chemotherapy, $16(17.8 \%)$ received postoperative radiotherapy and $9(10 \%)$ received postoperative chemoradiation. 63 patients (70\%) had recurrence and 57 patients $(63.3 \%)$ died during the follow-up. The estimated 1-, 3-, 5-year DFS and OS rates were 76\%, 48\%, 36\% and $89 \%, 56 \%$, 41\%, respectively. Median DFS was 31.7 months (range, 1.5 to 71.5 months). Median OS was 45.5 months (range, 2.6 to 71.5 months).

\section{Analysis of immunohistochemical parameters}

Representative images of neutrophil and CD8+ lymphocyte infiltration are shown in Figure 1. The median densities of intratumoral and peritumoral neutrophils were 18.5 cells/ HPF (range, 0-387 cells/HPF) and 19 cells/HPF (range, 0247 cells/HPF), respectively. The median densities of intratumoral and peritumoral CD8+ lymphocytes were 19 cells/ HPF (range, 0-122 cells/HPF) and 32 cells/HPF (range, 0200 cells/HPF), respectively. Infiltration of neutrophils or CD8+ lymphocyte was identified in both stroma and islet of the tumor, and there were no marked differences in neutrophil or CD8+ lymphocyte density between different intratumoral areas. The density of intratumoral neutrophils was not significantly different from that of peritumoral areas $(p=0.348)$. The density of intratumoral CD8+ lymphocytes was significantly lower than that of peritumoral area $(\mathrm{p}<0.001)$. The distribution of neutrophils was not associated with that of CD8+ lymphocytes. The density of intratumoral neutrophils were not significantly associated with that of intratumoral CD8+ lymphocytes $(r=0.005$, $\mathrm{p}=0.966$ ), and the density of neutrophils in peritumoral areas were not significantly correlated with that of CD8+ lymphocytes in peritumor $(\mathrm{r}=-0.127, \mathrm{p}=0.232)$, either. The density of intratumoral neutrophil was not significantly 
Table 1 Correlation of intratumoral CD66+ neutrophils, CD8+ lymphocytes and NLR with clinicopathological parameters

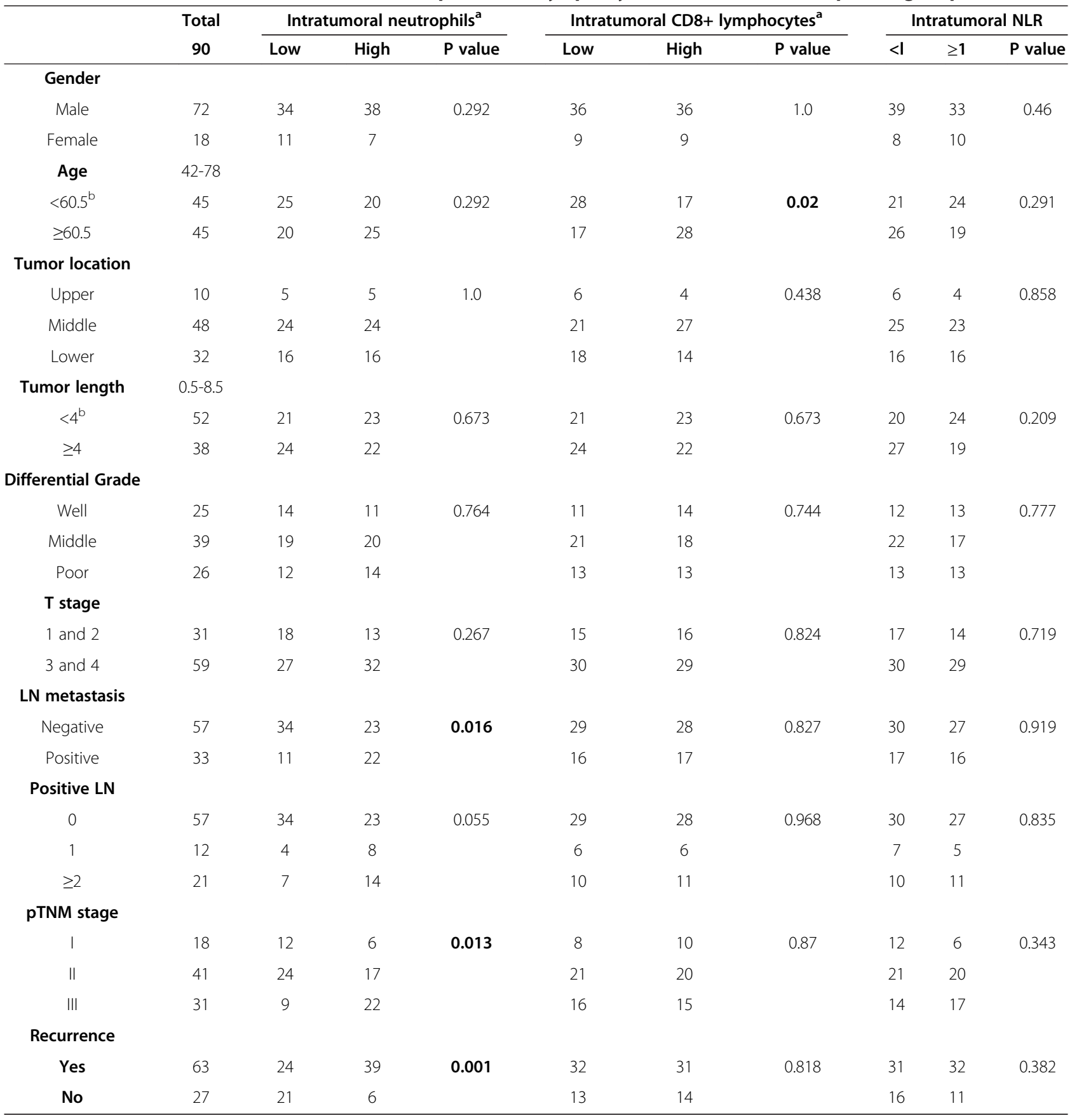

LN lymph node, pTNM pathological tumor node metastasis, NLR, CD66+ neutrophil-to-CD8+ lymphocyte ratio, values in bold signify $p<0.05$, ${ }^{\mathrm{a}}$ Grouped by median, ${ }^{\mathrm{b}}$ The median.

correlated with that in peritumor $(\mathrm{r}=0.202, \mathrm{p}=0.056)$. The density of intratumoral CD8+ lymphocytes was moderately correlated with that in peritumoral area $(r=0.434$, $\mathrm{p}<0.001$ ). For patients with lymph node metastasis, CD8+ lymphocyte density in intratumoral area was also significantly correlated with that in peritumor $(r=0.55$, $\mathrm{p}=0.001)$.

\section{Correlation of immunohistochemical parameters with} clinicopathological parameters

As is shown in Tables 1 and 2, increased intratumoral neutrophils was significantly associated with lymph node metastasis $(P=0.016)$, advanced pTNM stages $(P=0.013)$ and recurrence $(p=0.001)$. High intratumoral CD8+ lymphocytes were more frequently observed in elder patients $(\mathrm{p}=$ 


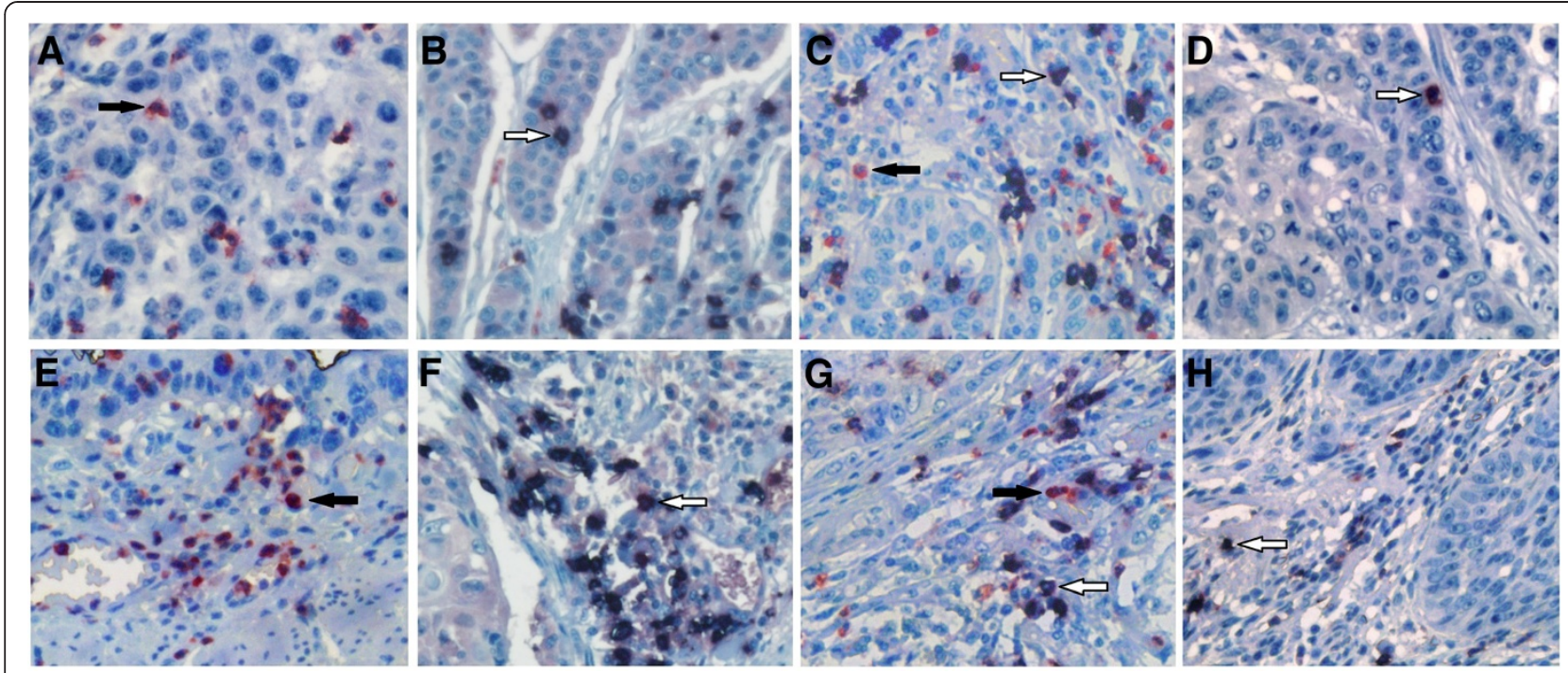

Figure 1 Representative examples of immunostaining of $C D 66 \mathrm{~b}+$ neutrophils (in red) or CD8+ lymphocytes (in black) from intratumoral $(A, B, C, D)$ or peritumoral $(E, F, G, H)$ areas of esophageal squamous cell carcinoma (x400). A, E infiltrated mainly by CD66b + neutrophils (black arrow); B, F infiltrated mainly by CD8+ lymphocytes (white arrow); C, G infiltrated by both CD66b + neutrophils and CD8+ lymphocytes; D, H infiltrated by low density of the inflammatory cells.

0.02). Intratumoral NLR was not associated with any of the clinicopathological parameters. Peritumoral neutrophil density was not associated with the studied clinicopathological parameters. Decreased peritumoral CD8+ lymphocyte density was more frequently observed in patients with single positive lymph node $(\mathrm{p}=0.045)$. Increased peritumoral NLR was associated with male gender $(p=0.009)$, advanced $T$ stages $(p<0.001)$, positive lymph node metastasis $(\mathrm{P}=0.041)$ and a trend towards advanced pathological stage $(\mathrm{p}=0.053)$.

\section{Survival analysis}

To identify variables of potential prognostic significance in the patients with ESCC, univariate and multivariate analyses were employed to investigate the impact of tumor-infiltrating neutrophils, CD8+ lymphocytes, NLR and other clinicopathological parameters on the prognosis of the 90 ESCC patients. In univariate analysis, increased intratumoral neutrophils were significantly associated with poor DFS $(\mathrm{p}<0.001)$ and OS $(\mathrm{p}<0.001)$ (Figure 2$)$. No prognostic significance was found for intratumoral and peritumoral CD8+ lymphocytes, intratumoral and peritumoral NLR and peritumoral neutrophils. The 5-year DFS and OS rates for patients with increased intratumoral CD66+ neutrophils were $20 \%$ and $26.7 \%$, compared with $51.1 \%$ and $55.5 \%$ for patients with decreased intratumoral CD66+ neutrophils, respectively. Clinicopathological factors significantly associated with short DFS were moderate differentiation, pT4 stage, lymph node metastasis and advanced pTNM stages. Variables significantly associated with poor OS were advanced T stages, lymph node metastasis, advanced pTNM stages and adjuvant radiation (Table 3 ). Multivariate analysis revealed that upper tumor location, advanced pTNM stages and increased intratumoral neutrophils were independently associated with decreased DFS and advanced pTNM stages, postoperative radiation, and increased intratumoral neutrophils were independent predictors for poor OS (Table 4).

\section{Discussion}

Inflammation seems to play a crucial role in cancer development by promoting or restraining progression, angiogenesis and metastasis, and impacting response to systemic therapies [20]. Neutrophils and lymphocytes, which constitute the predominant proportion of total circulating leukocytes, play a key role in host systemic immune response, but their effect on tumor progression should mostly be realized in the tumor microenvironment. In the present study, we utilized the methods of double staining immunohistochemistry to investigate the prognostic value of tumor-infiltrating neutrophils, CD8+ lymphocytes and NLR and to analyze the distribution of tumor-infiltrating neutrophils and CD8+ lymphocytes in ESCC treated by curative resection. Our study firstly established that increased intratumoral neutrophils were significantly correlated with lymph node metastasis and advanced pTNM stages, and increased peritumoral NLR was significantly associated with depth of invasion, lymph node metastasis and a trend towards advanced pathological stages in ESCC treated by curative resection. Moreover, univariate and multivariate analyses revealed that increased intratumoral neutrophils were a prognostic factor for poor DFS and 
Table 2 Correlation of peritumoral CD66+ neutrophils, CD8+ lymphocytes and NLR with clinicopathological parameters

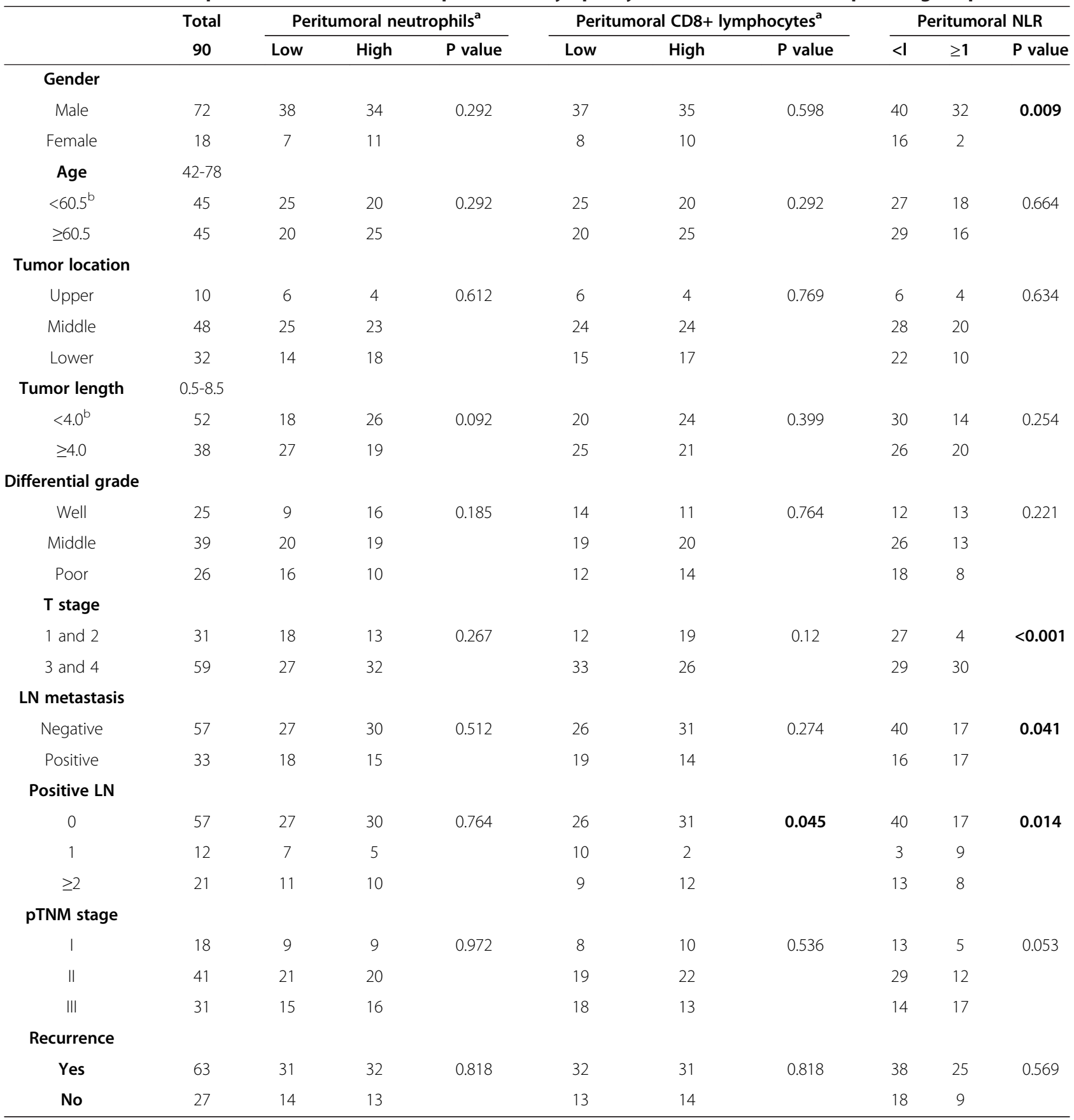

LN lymph node, pTNM pathological tumor node metastasis, NLR CD66+ neutrophil-to-CD8+ lymphocyte ratio, values in bold signify $\mathrm{p}<0.05$, ${ }^{\mathrm{a}}$ Grouped by median, ${ }^{\mathrm{b}}$ The median.

OS, independent of certain well-established clinical features, including depth of invasion, lymph node metastasis, and PTNM stages.

Neutrophils are short-lived white blood cells derived from bone marrow myeloid precursors. Attention has long been focused on their short-term antimicrobial and tissuedamaging function. However, growing evidence recently suggests that neutrophils play an important role in host's reaction to cancer [21]. The presence of tumor-associated neutrophils have been demonstrated to be associated with poor clinical outcomes of several malignancies including clear cell renal cell carcinoma [9], gastric cancer [22], colorectal cancer [11], and hepatocellular carcinoma [10]. The present study firstly investigated the clinical significance of tumor infiltrating neutrophils in ESCC. Our results revealed that intratumoral neutrophils be a significant 

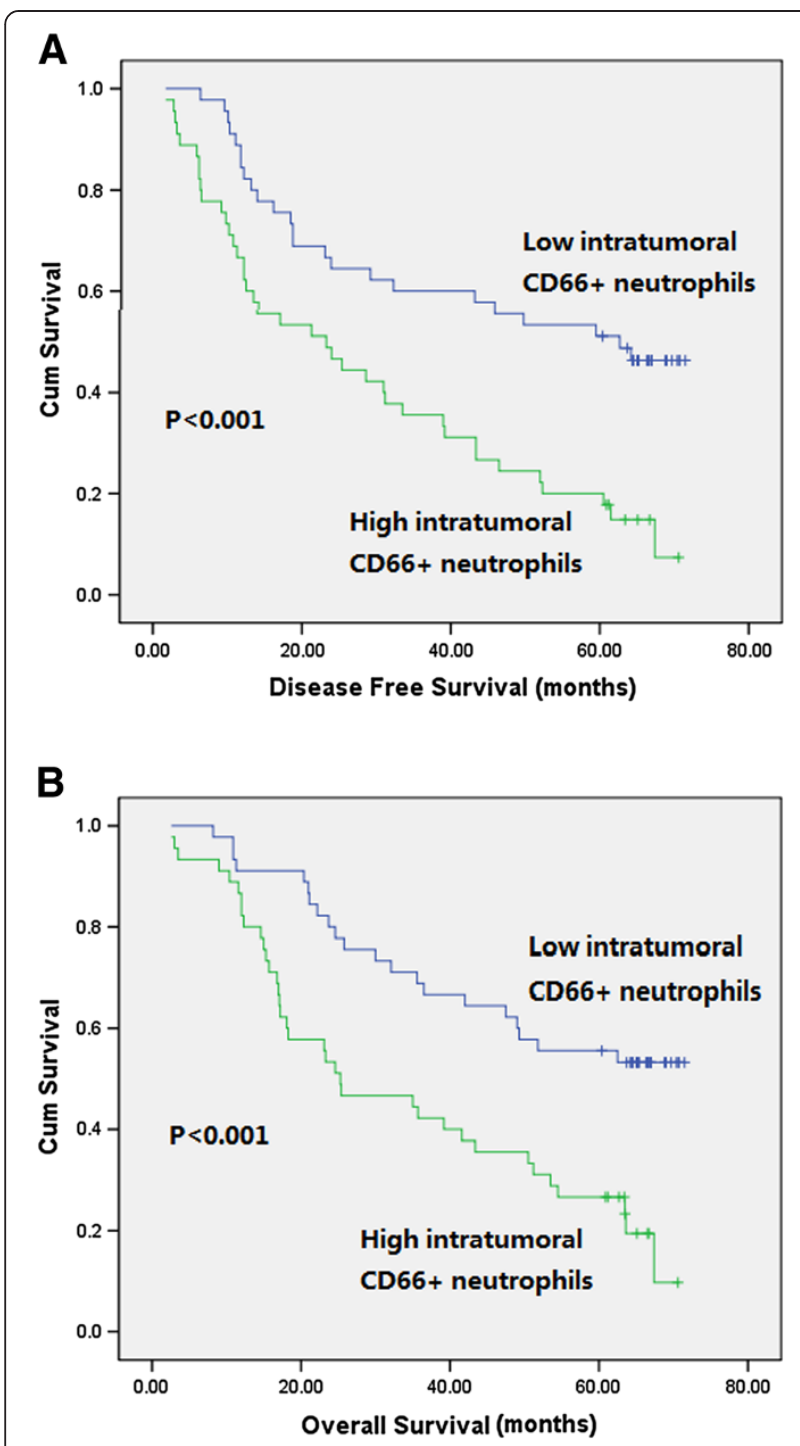

Figure 2 Kaplan-Meier analysis of intratumoral CD66b + neutrophils of 90 patients with esophageal squamous cell carcinoma. Increased intratumoral neutrophils were significantly associated with decreased disease-free survival (A) and overall survival (B).

prognostic factor in ESCC treated by curative surgery, which was consistent with the results of previous studies in other tumors.

Currently, the exact role of neutrophils in cancer initiation, progression and metastasis remains unclear. It has been hypothesized that tumor-associated neutrophils may have different states of differentiation which can thus take an anti-tumorigenic ("N1-phenotype") versus a pro-tumorigenic ("N2") phenotype [23]. The states of neutrophils in tumor tissue were recognized by expression of immuno-activating cytokines and chemokines, and capability of killing tumor cells. In models of lung cancer, infiltrating neutrophils are driven by TGF-b to acquire a protumor phenotype. The phagolysosomes of neutrophils contain several enzymes capable of reducing molecular oxygen into superoxide radicals. Reactive oxygen species from neutrophils were reported to exert both genotoxic effects and carcinogenesis [24]. Neutrophils could also release a group of proteinases including neutrophil elastase, matrix metalloproteinase- 8 , and matrix metalloproteinase- 9 to process and degrade a wide range of cytokines, chemokines, and their cognate receptors, in addition to their well-described ability to remodel extracellular matrix and enhance angiogenesis, which could promote the invasion and metastasis of cancer cells [25-27].

It is well recognized that cytotoxic CD8+ lymphocytes constitute one of the most important effector mechanisms of anti-tumor immunity [28]. In both animal models and humans, CD8+ lymphocytes have been shown to play an important role in the host's defense against malignancies, and most cancer vaccine strategies have focused on the induction of effector CD8+ lymphocytes that kill tumor cells [29]. It has been shown that a high infiltration of CD8+ lymphocytes be associated with good clinical outcome in several types of human cancer, including cancer of esophagus [30-33]. However, other studies did not show the clinical significance of tumor infiltrated CD8+ lymphocytes [34]. The inconsistent results in different studies could be explained by discrepancies in sample size, study population, treatment modality, and pathological type. In our study, we did not found any association between intratumoral or peritumoral CD8+ lymphocyte infiltration and clinicopathological parameter such as depth of invasion, pathological stages, or clinical outcome. Only when analyzed by positive lymph node number, low peritumoral CD8+ lymphocytes were associated with one lymph node metastasis. Tumor-infiltrating CD8+ lymphocytes could not predict progression or prognosis in ESCC treated by curative resection. Our results indicated that the antitumor effect of CD8+ lymphocytes could be partly impaired in the microenvironment of esophageal squamous cell carcinoma, especially in the intratumoral area. The impairment may be mediated by the following mechanisms: immunosuppressing factors released by tumor cells, deficient tumor antigen presentation by dendritic cells, reduced production of costimulating cytokines by helper CD4+ T-cells, and recruitment of regulatory immune cells by tumor cells [35].

Recently, great interests have been generated in elucidating the role of cancer inflammation in predicting disease load and prognosis. Blood neutrophil to lymphocyte ratio has been found to be associated with disease progression in a number of malignancies [36]. However, the systemic immune cells must infiltrate the tumor tissue to function their antitumor or protumor effect. Cancer cells recruit inflammatory cells, including neutrophils, which can suppress the action of cytotoxic lymphocytes. 
Table 3 Univariate analysis of clinicopathological and immunohistochemical parameters

\begin{tabular}{|c|c|c|c|c|}
\hline \multirow[t]{2}{*}{ Univariate analysis } & \multicolumn{2}{|c|}{ Disease-free survival } & \multicolumn{2}{|c|}{ Overall survival } \\
\hline & HR $(95 \% \mathrm{Cl})$ & $P$ value & HR $(95 \% \mathrm{Cl})$ & $P$ value \\
\hline Gender & $1.182(0.64-2.185)$ & 0.593 & $1.154(0.608-2.19)$ & 0.661 \\
\hline Age & $1.092(0.665-1.793)$ & 0.727 & $1.27(0.753-2.144)$ & 0.37 \\
\hline \multicolumn{5}{|l|}{ Tumor location } \\
\hline Upper & Ref. & 0.213 & Ref. & 0.6 \\
\hline Middle & $0.517(0.2554-1.202)$ & 0.083 & $0.653(0.286-1.493)$ & 0.312 \\
\hline Lower & $0.553(0.545-1.608)$ & 0.135 & $0.698(0.296-1.65)$ & 0.413 \\
\hline Tumor length & $1.172(0.715-1.923)$ & 0.529 & $1.343(0.797-2.262)$ & 0.269 \\
\hline \multicolumn{5}{|l|}{ Differential Grade } \\
\hline Well & Ref. & 0.095 & Ref. & 0.17 \\
\hline Moderate & $1.947(1.045-3.629)$ & 0.036 & $1.881(0.971-3.645)$ & 0.061 \\
\hline Poor & $1.334(0.666-2.672)$ & 0.417 & 1.449 (0.697-3.015) & 0.321 \\
\hline \multicolumn{5}{|l|}{ T stage } \\
\hline $\mathrm{T} 1$ & Ref. & 0.121 & Ref. & 0.026 \\
\hline $\mathrm{T} 2$ & $1.359(0.539-3.454)$ & 0.519 & $1.425(0.477-4.253)$ & 0.526 \\
\hline T3 & $1.954(0.871-4.385)$ & 0.104 & $2.706(1.061-6.9)$ & 0.037 \\
\hline $\mathrm{T} 4$ & $3.328(1.113-9.951)$ & 0.031 & $4.618(1.404-15.196)$ & 0.012 \\
\hline $\mathrm{LN}$ metastasis & $2.506(1.51-4.157)$ & $<0.001$ & $2.469(1.455-4.188)$ & 0.001 \\
\hline \multicolumn{5}{|l|}{ Positive LN number } \\
\hline 0 & Ref. & 0.001 & Ref. & 0.002 \\
\hline 1 & $3.479(1.756-6.89)$ & $<0.001$ & $3.01891 .491-6.112$ & 0.002 \\
\hline$\geq 2$ & $2.117(1.176-3.812)$ & 0.012 & 2.20391.195-4.063 & 0.011 \\
\hline \multicolumn{5}{|l|}{ pTNM stage } \\
\hline 1 & Ref. & $<0.001$ & Ref. & $<0.001$ \\
\hline$\|$ & $2.03(0.919-4.482)$ & 0.08 & $2.334(0.951-5.729)$ & 0.064 \\
\hline III & $4.365(1.962-9.713)$ & $<0.001$ & $5.254(2.139-12.91)$ & $<0.001$ \\
\hline \multicolumn{5}{|l|}{ Treatment regimens } \\
\hline Surgery alone & Ref. & 0.081 & Ref. & 0.063 \\
\hline Adjuvant chemotherapy & $0.839(0.354-1.993)$ & 0.692 & $0.747(0.291-1.919)$ & 0.545 \\
\hline Adjuvant radiation & $1.821(0.961-3.451)$ & 0.066 & $1.99(1.04-3.807)$ & 0.038 \\
\hline Adjuvant chemoradiation & $2.152(0.992-4.67)$ & 0.053 & $2.054(0.902-4.676)$ & 0.086 \\
\hline \multicolumn{5}{|l|}{ Immunohistochemical markers } \\
\hline Intratumoral neutrophils & $2.505(1.496-4.196)$ & $<0.001$ & $2.559(1.487-4.406)$ & $<0.001$ \\
\hline Intratumoral CD8+ lymphocytes & $0.896(0.546-1.471)$ & 0.665 & $0.959(0.57-1.613)$ & 0.875 \\
\hline Intratumoral NLR & $1.279(0.78-2.097)$ & 0.329 & $1.316(0.783-2.214)$ & 0.3 \\
\hline Peritumoral neutrophils & $1.035(0.63-1.7)$ & 0.893 & 1.119 (0.664-1.886) & 0.672 \\
\hline Peritumoral CD8+ lymphocytes & $0.952(0.581-1.561)$ & 0.846 & $1.032(0.613-1.735)$ & 0.907 \\
\hline Peritumoral NLR & $1.306(0.788-2.165)$ & 0.3 & $1.292(0.761-2.195)$ & 0.343 \\
\hline
\end{tabular}

LN lymph node, pTNM pathological tumor node metastasis, NLR CD66+ neutrophil-to-CD8+ lymphocyte ratio, HR hazard ratio, Cl confidence interval, Ref. reference, Values in bold signify $\mathrm{p}<0.05$.

Cancer cells themselves may secrete various molecules to induce a tumor facilitating cytotoxic lymphocytes. Currently, evidence is limited about the prognostic effect of tumor-infiltrating NLR in malignancies. Marius et al. [19] assessed the prognostic impact of intratumoral NLR in resectable non-small cell lung cancer and found it as an independent prognostic factor for a high rate of disease recurrence and poor survival. Another study showed that elevated intratumoral NLR was significantly associated with decreased DFS and OS in hepatocellular carcinoma 
Table 4 Independent predictors for survival by multivariate analysis

\begin{tabular}{lccc}
\hline Multivariate analysis & Category compared & HR (95\% Cl) & P value \\
\hline Disease-free survival & & & \\
Tumor location & Upper vs. middle and lower & $0.474(0.228-0.987)$ & 0.046 \\
PTNM stage & I, II vs. III & $1.892(1.104-3.241)$ & 0.02 \\
Intratumoral neutrophils & $<18.5$ vs. $\geq 18.5$ & $2.174(1.249-3.784)$ & 0.006 \\
Overall survival & I, II vs. III & $2.122(1.205-3.736)$ & 0.009 \\
pTNM stage & Surgery alone vs. Adjuvant radiation & $1.855(1.061-3.244)$ & 0.03 \\
Treatment regimens & $<18.5$ vs. $\geq 18.5$ & $1.858(1.038-3.325)$ & 0.037 \\
Intratumoral neutrophils & &
\end{tabular}

pTNM pathological tumor node metastasis, HR hazard ratio, $\mathrm{Cl}$ confidence interval.

following resection [10]. In the present study, we firstly analyzed the clinical significance of both intratumoral and peritumoral NLRs in ESCC treated by curative surgery. Although no prognostic effect of intratumoral or peritumoral NLR was shown, we interestingly found that increased peritumoral NLR was associated with depth of invasion, lymph node metastasis and a trend towards advanced pathological stages. In the peritumoral region of ESCC, infiltrating neutrophils may suppress the cytotoxic response of CD8+ lymphocytes and thereby allow tumor cells to evade immune surveillance. The results are in agreement with recent findings showing that, in mice, naturally activated neutrophils in the tumor microenvironment were mainly of the polarized $\mathrm{N} 2$ phenotype promoting tumor progression, partly associated with suppression of CD8+ lymphocytes [23]. However, this does not preclude the possibility that peritumoral microenvironment with high neutrophil and low CD8+ lymphocyte infiltration is more suitable for tumor invasion and both immune cells do not act on each other directly.

Human tumor tissue can be anatomically classified into areas of intratumor and peritumor, each with distinct compositions and functional properties. The density of intratumoral neutrophil was not significantly different from or correlated with that of peritumoral area, whereas the density of intratumoral CD8+ lymphocytes was significantly lower than and moderately correlated with that of peritumoral area. Infiltrating of intratumoral CD8+ lymphocyte may partly determined by recruitment of the lymphocytes in the peritumoral region. Intratumoral environment usually exhibited a generalized immunosuppressive status, resulting in a relatively low density of intratumoral cytoxic CD8+ lymphocytes. We observed great discrepancies in the amount of both neutrophil and CD8+ lymphocyte infiltration between different patients. The mechanism why patients may be associated with mild or extensive level of inflammatory cells remains unclear. In addition, it has been found that the density of intratumoral neutrophils was significantly correlated with that of CD8+ lymphocytes in resected hepatocellular cell carcinoma [10]. Neutrophil depletion could result in more activated CD8+ lymphocytes intratumorally [23]. However, in our study, neither the distribution nor the density of neutrophils was correlated with that of CD8+ lymphocytes in ESCC, which suggest that the recruitment of the two types of inflammatory cells may represent different characteristic of ESCC that do not correlated directly.

\section{Conclusions}

In conclusion, we showed that increased intratumoral CD $66+$ neutrophils were an independent prognostic factor for poor DFS and OS in ESCC treated by curative surgery. Moreover, we demonstrated that increased peritumoral NLR was a predictor for advanced $T$ stages, lymph node metastasis and a trend towards advanced disease stages. This indicated that the progression of ESCC may be governed at least in part by the state of the local innate immune response. This might also aid the clinician to select a suitable therapy for the individual patients, e.g. favoring a more aggressive regimen in tumors with an increased intratumoral neutrophils or misbalance of peritumoral neutrophil and CD8+ lymphocytes. Moreover, Immunotherapy targeted to the above predictors may also help improve the prognosis of esophageal cancer.

\section{Abbreviations}

ESCC: Esophageal squamous cell carcinoma; NLR: Neutrophils and neturophil-toCD8+ lymphocyte ratio; DFS: Disease-free survival; OS: Overall survival.

\section{Competing interests}

The authors declare that they have no competing interest.

\section{Authors' contributions}

WJB carried out experiments, analyzed the data, and participated in the study design and manuscript writing. JYB and WNN contributed to data analyzing; ZXM, TBX and ZGY collected data, and participated in experiment performance. CYF designed experiments and wrote the manuscript. All authors read and approved the final version of the manuscript.

\section{Acknowledgements}

The work was supported by Science and Technology plan project of Shandong province 2012GSF11852, Natural Science Fund of Shandong Province 2010ZRE27212 and Jinan Scientific project 201202026. We should thank Junhui Zhen (pathologist in Department of Pathology, Qilu Hospital of Shandong University, China) for her expert suggestions and technical assistance.

Received: 8 August 2013 Accepted: 27 November 2013

Published: 7 January 2014 


\section{References}

1. Jemal A, Bray F, Center MM, Ferlay J, Ward E, Forman D: Global cancer statistics. Ca Cancer J Clin 2011, 61:69-90.

2. MAO YS, HE J, Cheng GY: Current status of surgical management of esophageal cancer in china and the future strategy. Zhonghua Zhong Liu Za Zhi 2010, 32:401-404.

3. Kleinberg L, Forastiere AA: Chemoradiation in the management of esophageal cancer. J Clin Oncol 2007, 25:4110-4117.

4. Cheng TH, Hsu PK, Li AF, Hung IC, Huang MH, Hsu HS: Correlation of p53, MDM2 and p14(ARF) protein expression in human esophageal squamous cell carcinoma. J Cancer Res Clin Oncol 2009, 135:1577-1582.

5. Zitvogel L, Tesniere A, Kroemer G: Cancer despite immunosurveillance: immunoselection and immunosubversion. Nat Rev Immunol 2006, 6:715-727.

6. Bremnes RM, Al-Shibli K, Donnem T, Sirera R, Al-Saad S, Andersen S, Stenvold H, Camps C, Busund LT: The role of tumor-infiltrating immune cells and chronic inflammation at the tumor site on cancer development, progression, and prognosis: emphasis on non-small cell lung cancer. J Thorac Oncol 2011, 6:824-833.

7. Gregory AD, Houghton AM: Tumor-associated neutrophils: new targets for cancer therapy. Cancer Res 2011, 71:2411-2416.

8. Hofman PM: Pathobiology of the neutrophil-intestinal epithelial cell interaction: role in carcinogenesis. World J Gastroenterol 2010, 16:5790-5800.

9. Jensen HK, Donskov F, Marcussen N, Nordsmark M, Lundbeck F, Von Der Maase H: Presence of intratumoral neutrophils is an independent prognostic factor in localized renal cell carcinoma. J Clin Oncol 2009, 27:4709-4717.

10. Li YW, Qiu SJ, Fan J, Zhou J, Gao Q, Xiao YS, Xu YF: Intratumoral neutrophils: a poor prognostic factor for hepatocellular carcinoma following resection. J Hepatol 2011, 54:497-505.

11. Rao HL, Chen JW, Li M, Xiao YB, Fu J, Zeng YX, Cai MY, Xie D: Increased intratumoral neutrophil in colorectal carcinomas correlates closely with malignant phenotype and predicts patients' adverse prognosis. Plos One 2012, 7:E30806.

12. Coussens LM, Werb Z: Inflammation and cancer. Nature 2002, 420:860-867.

13. Mcmillan DC, Canna K, Mcardle CS: Systemic inflammatory response predicts survival following curative resection of colorectal cancer. Br J Surg 2003, 90:215-219.

14. Zahorec R: Ratio of neutrophil to lymphocyte counts-rapid and simple parameter of systemic inflammation and stress in critically ill. Bratis/ Lek Listy 2001, 102:5-14.

15. Mcmillan DC, Wotherspoon HA, Fearon KC, Sturgeon C, Cooke TG, Mcardle CS: A prospective study of tumor recurrence and the acute-phase response after apparently curative colorectal cancer surgery. Am J Surg 1995, 170:319-322.

16. Sharaiha RZ, Halazun K, Mirza F, Port JL, Lee PC, Neugut Al, Altorki NK, Abrams JA: Elevated preoperative neutrophil:lymphocyte ratio as a predictor of postoperative disease recurrence in esophageal cancer. Ann Surg Oncol 2011, 18:3362-3369.

17. Hsu PK, Wu YC, Chou TY, Huang CS, Hsu WH: Comparison of the 6th and 7th editions of the American joint committee on cancer tumor-nodemetastasis staging system in patients with resected esophageal carcinoma. Ann Thorac Surg 2010, 89:1024-1031.

18. ANDO N, lizuka T, Ide H, Ishida K, Shinoda M, Nishimaki T, Takiyama W, Watanabe H, Isono K, Aoyama N, et al: Surgery plus chemotherapy compared with surgery alone for localized squamous cell carcinoma of the thoracic esophagus: a Japan clinical oncology group study-JCOG9204. J Clin Oncol 2003, 21:4592-4596.

19. Ilie M, Hofman V, Ortholan C, Bonnetaud C, Coelle C, Mouroux J, Hofman P: Predictive clinical outcome of the intratumoral CD66b-positive neutrophil-toCD8-positive T-cell ratio in patients with resectable nonsmall cell lung cancer. Cancer 2012, 118:1726-1737.

20. Mantovani A, Allavena P, Sica A, Balkwill F: Cancer-related inflammation. Nature 2008, 454:436-444.

21. Houghton AM: The paradox of tumor-associated neutrophils: fueling tumor growth with cytotoxic substances. Cell Cycle 2010, 9:1732-1737.

22. Zhao JJ, Pan K, Wang W, Chen JG, Wu YH, Lv L, Li JJ, Chen YB, Wang DD, Pan QZ, et al: The prognostic value of tumor-infiltrating neutrophils in gastric adenocarcinoma after resection. Plos One 2012, 7:E33655.

23. Fridlender ZG, Sun J, Kim S, Kapoor V, Cheng G, Ling L, Worthen GS, Albelda SM: Polarization of tumor-associated neutrophil phenotype by TGF-beta: "N1" versus "N2" TAN. Cancer Cell 2009, 16:183-194.
24. Gungor N, Knaapen AM, Munnia A, Peluso M, Haenen GR, Chiu RK Godschalk RW, VAN Schooten FJ: Genotoxic effects of neutrophils and hypochlorous acid. Mutagenesis 2010, 25:149-154.

25. Houghton AM, Rzymkiewicz DM, JI H, Gregory AD, EGEA EE, Metz HE, Stolz DB, Land SR, Marconcini LA, Kliment CR, et al: Neutrophil elastase-mediated degradation of IRS-1 accelerates lung tumor growth. Nat Med 2010, 16:219-223.

26. Coussens LM, Tinkle CL, Hanahan D, Werb Z: MMP-9 supplied by bone marrow-derived cells contributes to skin carcinogenesis. Cell 2000 103:481-490.

27. Gutierrez-Fernandez A, Fueyo A, Folgueras AR, Garabaya C, Pennington CJ, Pilgrim S, Edwards DR, Holliday DL, Jones JL, SPAN PN, et al: Matrix metalloproteinase-8 functions as a metastasis suppressor through modulation of tumor cell adhesion and invasion. Cancer Res 2008, 68:2755-2763.

28. Shanker A, Verdeil G, Buferne M, Inderberg-Suso EM, Puthier D, Joly F, Nguyen C, Leserman L, Auphan-Anezin N, Schmitt-Verhulst AM: CD8 T cell help for innate antitumor immunity. J Immunol 2007, 179:6651-6662.

29. Williams MA, Bevan MJ: Effector and memory CTL differentiation. Annu Rev Immunol 2007, 25:171-192.

30. Gooden MJ, DE Bock GH, Leffers N, Daemen T, Nijman HW: The prognostic influence of tumour-infiltrating lymphocytes in cancer: a systematic review with meta-analysis. Br J Cancer 2011, 105:93-103.

31. Ikeguchi M, Saito H, Katano K, Tsujitani S, Maeta M, Kaibara N: Correlation between the lymphocytic infiltration of tumors and the proliferative activity of cancer cells from surgically treated esophageal carcinoma. Oncology 1997, 54:311-317.

32. Schumacher K, Haensch W, Roefzaad C, Schlag PM: Prognostic significance of activated $\mathrm{CD} 8(+) \mathrm{T}$ cell infiltrations within esophageal carcinomas. Cancer Res 2001, 61:3932-3936.

33. Cho $Y$, Miyamoto M, Kato $K$, Fukunaga A, Shichinohe T, Kawarada $Y$, Hida $Y$, Oshikiri T, Kurokawa T, Suzuoki M, et al: CD4+ And CD8+ T cells cooperate to improve prognosis of patients with esophageal squamous cell carcinoma. Cancer Res 2003, 63:1555-1559.

34. Zingg U, Montani M, Frey DM, Dirnhofer S, Esterman AJ, Went P, Oertli D: Tumour-infiltrating lymphocytes and survival in patients with adenocarcinoma of the oesophagus. Eur J Surg Oncol 2010, 36:670-677.

35. Prado-Garcia H, Romero-Garcia S, Aguilar-Cazares D, Meneses-Flores M, lopez-gonzalez JS: Tumor-induced CD8+ T-cell dysfunction in lung cancer patients. Clin Dev Immunol 2012, 2012:741741

36. Roxburgh C, Mcmillan D: Role of systemic inflammatory response in predicting survival in patients with primary operable cancer. Future Oncol 2010, 6:149-163.

doi:10.1186/1479-5876-12-7

Cite this article as: Wang et al:: The clinical significance of tumorinfiltrating neutrophils and neutrophil-to-CD8+ lymphocyte ratio in patients with resectable esophageal squamous cell carcinoma. Journal of Translational Medicine 2014 12:7.

\section{Submit your next manuscript to BioMed Central and take full advantage of:}

- Convenient online submission

- Thorough peer review

- No space constraints or color figure charges

- Immediate publication on acceptance

- Inclusion in PubMed, CAS, Scopus and Google Scholar

- Research which is freely available for redistribution 